\title{
Evaluation of the Long-Term Changes in Fatigue of Cancer Survivors Who Received Outpatient Chemotherapy in the Area Affected by the Great East Japan Earthquake
}

\author{
Daisuke Sato \\ Faculty of Health Sciences, Komatsu University, Ishikawa, Japan \\ Email: daisuke.sato@komatsu-u.ac.jp
}

How to cite this paper: Sato, D. (2021) Evaluation of the Long-Term Changes in Fatigue of Cancer Survivors Who Received Outpatient Chemotherapy in the Area Affected by the Great East Japan Earthquake. Open Journal of Nursing, 11, 566-577. https://doi.org/10.4236/ojn.2021.117048

Received: April 27, 2021

Accepted: July 16, 2021

Published: July 19, 2021

Copyright (อ 2021 by author(s) and Scientific Research Publishing Inc. This work is licensed under the Creative Commons Attribution International License (CC BY 4.0).

http://creativecommons.org/licenses/by/4.0/

\begin{abstract}
Objective: The aim of the study is to evaluate the long-term changes in fatigue of cancer patients receiving outpatient chemotherapy after a disaster such as the Great Earthquake. Methods: From 2012 to 2014, we measured subjective fatigue, autonomic function, and physical activity with reference to the criteria for chronic fatigue syndrome. Results: The participants were 13, 9 males $(69.2 \%)$ and 4 females $(30.8 \%)$. The most common cancer among the participants was lung cancer 4 patients (30.8\%). In 2012, eight patients $(61.6 \%)$ received anti-cancer drugs and three $(23.0 \%)$ received the drug by intravenous drip and oral administration. Physical fatigue $(\mathrm{p}=0.004)$, mental fatigue ( $p=0.002)$, comprehensive fatigue $(p=0.012)$, and comprehensive evaluations ( $p=0.032)$ were significantly different. Significant differences in the autonomic nervous function were observed during the three years from 2012 to $2014(\log \mathrm{LF}[\mathrm{p}=0.017], \log \mathrm{HF}[\mathrm{p}=0.023]$ and $\log \mathrm{LF} / \mathrm{HF}$ [p = 0.039]). Similar to Subjective fatigue, Significant differences in physical activity were observed during the three years from 2012 to 2014 (DA [p = 0.042], TST [p = 0.037], SE [p = 0.018], AW [p=0.023] and Naps [p=0.014]). Conclusions: The participants fatigue worsened at one time, but showed improvement over time. In the event of an emergency such as a disaster, it is necessary to evaluate the physical and mental condition based on objective indicators of cancer patients receiving anti-cancer drug treatment and provide nursing support.
\end{abstract}

\section{Keywords}

Cancer Survivors, Outpatient Chemotherapy, Fatigue, The Great East Japan 
Earthquake

\section{Introduction}

Treatments surrounding cancer patients in Japan are shifting from wards to home care due to shorter hospital stays. The background of this is the development of cancer drug therapy, social demands for cancer care, and medical economics reasons [1]. Cancer patients need to be able to continue their outpatient treatment while living their normal and social lives, and to maintain and improve their quality of life. Post-disaster medical institutions' top priority is to triage, prioritize and provide treatment to patients in need. Molecular-targeted drugs and immune checkpoint inhibitors, hormone therapy cause fatigue, nausea and diarrhea, and fever due to neutropenia. Therefore, side effect management needs to be devised according to the patient's self-care ability and living environment, such as observation contents and coping methods, according to the characteristics of the drug.

Japan is a country with many natural disasters such as earthquakes and typhoons. In the event of a large-scale disaster, many emergency patients are transported to hospitals and many medical staff are assigned.

For cancer patients who were scheduled for outpatient chemotherapy, treatment of emergency patients was prioritized and treatment was postponed [2]. Cancer patients are worried that the disease will progress by postponing treatment. Before and after the disaster, they were stressed and the rate of recognizing subjective symptoms was increasing [3]. They think that one of the reasons is that they lost their families and homes due to the disaster and became mentally unstable, and the living environment changed drastically [3] [4] [5].

Previously, I used a system that objectively assesses the fatigue of cancer patients undergoing outpatient chemotherapy to determine the nursing support needed during a disaster [2]. As a result, the subjective malaise of cancer patients and the objective evaluation did not correlate and did not match. Cancer patients in the affected areas are affected by the stress of disasters and treatments, and are physically and mentally exhausted. An environment exposed to excessive tension and stress controls the sympathetic nervous system, affects sleep, increases the risk of malaise, weakens immunity and increases the risk of infection [6]. Cancer patients and cancer survivors living in the affected areas may experience chronic fatigue and put a strain on their physical and mental health. If this condition persists, it may increase the risk of worsening cancer status, chronic fatigue syndrome, sudden death, death from overwork, cardiovascular disease, and mental illness. It takes a long time to recover from a disaster. Chemotherapy will also continue for a long period of time. Therefore, it is important to support fatigue, which is one of the effects of stress on cancer patients in the disaster area.

Previous studies have investigated changes over time in a single year. The aim study is to evaluate the long-term changes in fatigue of cancer patients receiving 
outpatient chemotherapy after a disaster such as the Great Earthquake.

\section{Methods}

\subsection{The Type of Study Design}

Descriptive study designs.

\subsection{Study Period}

The study period was three years from 2012 to 2014, and data were collected in September of each year.

\subsection{Participants}

In 2012, Cancer outpatients undergoing chemotherapy at a general hospital in the area affected by the Great East Japan Earthquake were randomly selected as participants. A survey was conducted at the selected hospital for 3 years. At the same time, those who consented to the research cooperation for 3 years were selected as the research subjects.

The inclusion criteria for participants are as follows: 1) ages 20 - 75 years. 2) Diagnosed with cancer and receiving treatment at an outpatient chemotherapy center. 3) The type of cancer and the administration method of the anticancer drug (oral or intravenous drip) do not matter. 4) It does not matter whether it is the first time or a recurrence. 5) No mental illness. 6) Those who have consented to the research and obtained the permission of the doctor.

The exclusion criteria for participants are as follows: 1) having active, serious physical disease that affects household and light work 2) inability to understand Japanese; 3) currently undergoing follow-up and treatment in a psychiatry department or by other mental health professionals; 4) judged inappropriate for participation by the researchers (e.g., identity theft, duplicate entry, and so on).

\section{Participant Characteristics}

This study received consent from 22 patients to participate in the study in 2012, but there were some who dropped out because of death and loss of contact during the process. Ultimately, 13 subjects who participated in the study for 3 years were analyzed. Participant flow diagram is shown in Figure 1.

The study participants consisted of 13 cancer outpatients ( 9 men [69.2\%] and 4 women [30.8\%]). The most common of the participants was lung cancer (4 patients [30.8\%]). In 2012, Eight patients (61.6\%) were treated by intravenous infusion and three $(23.0 \%)$ were treated by both intravenous and oral administration. In 2013 and 2014, 3 (25.4\%) and 1 (7.7\%) continued treatment, respectively. The percentage of " 0 " in PS increased with the aging. The damage to their houses was completely destroyed in 2 participants (15.4\%) and partial damage in 4 participants (30.8\%). The number of temporary housing residents was 5 (38.5\%) in 2012, but decreased to $2(15.4 \%)$ in 2014. Participant characteristics are shown in Table 1. 
Cancer outpatients undergoing chemotherapy at a clinic in a general hospital in an area affected by the Great East Japan Earthquake 2012: $(\mathrm{n}=22)$

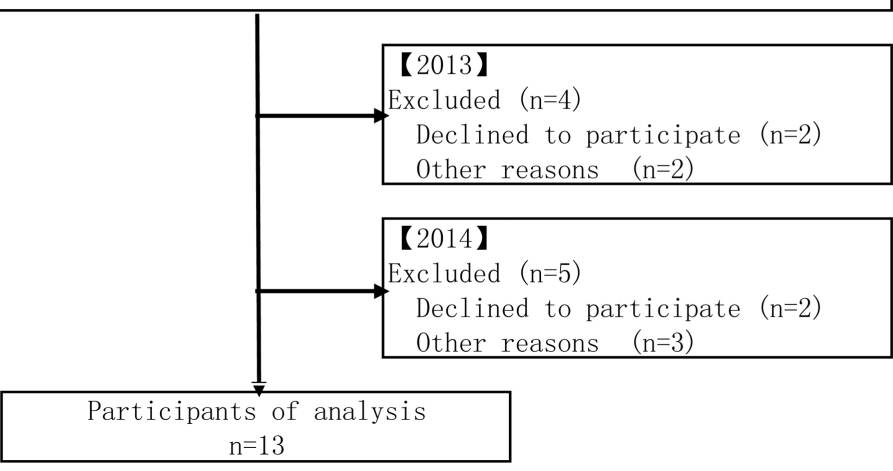

Figure 1. Participant flow diagram.

Table 1. Characteristics of patients $(n=13)$.

\begin{tabular}{|c|c|c|c|c|c|}
\hline \multirow{2}{*}{ Item } & & & 2012 & 2013 & 2014 \\
\hline & & \multicolumn{4}{|c|}{$\mathrm{n}(\%)$} \\
\hline Average age & & & $63.2 \pm 10.3$ & $64.2 \pm 10.3$ & $65.2 \pm 10.3$ \\
\hline \multirow{2}{*}{ Gender } & Men & $9(69.2)$ & & & \\
\hline & Women & $4(30.8)$ & & & \\
\hline \multirow{5}{*}{ Type of cancer } & Lung & $4(30.8)$ & & & \\
\hline & Rectum & $2(15.4)$ & & & \\
\hline & Breast & $3(23.0)$ & & & \\
\hline & Stomach & $2(15.4)$ & & & \\
\hline & Prostate & $2(15.4)$ & & & \\
\hline Surgery history & Yes & $6(46.2)$ & & & \\
\hline \multirow{3}{*}{$\begin{array}{l}\text { Administration method } \\
\text { of anticancer drug }\end{array}$} & Drip & & $8(61.6)$ & $2(15.4)$ & 0 \\
\hline & internal use & & $2(15.4)$ & $1(7.7)$ & $1(7.7)$ \\
\hline & Both & & $3(23.0)$ & 0 & 0 \\
\hline Spouse & Exist & & $7(53.8)$ & $6(46.2)$ & $6(46.2)$ \\
\hline Working & Yes & & $4(30.8)$ & $3(23.0)$ & $3(23.0)$ \\
\hline \multirow{3}{*}{ Performance Status } & 0 & & $3(23.0)$ & $6(46.2)$ & $7(53.8)$ \\
\hline & 1 & & $6(46.2)$ & $5(38.4)$ & $5(38.4)$ \\
\hline & 2 & & $4(30.8)$ & $2(15.4)$ & $1(7.8)$ \\
\hline \multirow{2}{*}{ Damage to houses } & Complete destruction & $2(15.4)$ & & & \\
\hline & Partial destruction & $4(30.8)$ & & & \\
\hline Temporary housing & Entering & & $5(38.5)$ & $5(38.4)$ & $2(15.4)$ \\
\hline
\end{tabular}

\subsection{Content of Evaluations}

In this study, patient evaluation was analyzed based on individual attributes and objective data on autonomic nerves and sleep, which are diagnostic criteria for chronic fatigue syndrome [7] [8] [9]. Among the auxiliary items required for the diagnosis of chronic fatigue syndrome, evaluation of autonomic nervous function and physical activity (evaluation of sleep time and average arousal activity) 
was selected. For personal attributes, we investigated age, gender, cancer type, cancer treatment drug, marital status, employment status, performance status (PS), cancer stage, housing damage status, and temporary housing status. Autonomic nervous function was evaluated by measuring acceleration pulse waves. A pulse wave meter (Altet 2; U-Medica, Osaka, Japan) was used for the acceleration pulse wave measurement. The low frequency (LF) component ( 0.04 to 0.15 $\mathrm{Hz})$ is an index of sympathetic nerve function, and the high frequency (HF) component $(0.15$ to $0.40 \mathrm{~Hz})$ is an index of parasympathetic nerve function [10]. Relative sympathetic function was calculated based on the LF/HF ratio, with less than 2 being good. A questionnaire survey was conducted to evaluate the subjective malaise of the participants. The reliability and validity of the questionnaire have been verified by the Ministry of Health, Labor and Welfare [7] [8] [9]. This questionnaire consists of 10 items of physical fatigue ( 40 points), 10 items of mental fatigue (40 points), and 20 items of total fatigue ( 80 points). Items of physical and mental malaise are scored on a scale of none (0) to very strong (4) and evaluated on a total basis as follows: physical fatigue for men, 0 - 7 points (good), 8 - 11 points (attention), $\geq 12$ points (careful); physical fatigue for women, 0 - 8 points (good), 9 - 13 points (attention), $\geq 14$ points (careful); mental fatigue for men, 0 - 9 points (good), $10-12$ points (attention), $\geq 13$ points (careful); for women, $0-10$ points (good), $11-15$ points (attention), $\geq 16$ points (careful). Overall fatigue is the sum of the physical and mental fatigue scores: for men, 0 - 16 points (good), $17-22$ points (attention), $\geq 23$ points (careful); for women, 0 - 19 points (good), $20-28$ points (attention), and $\geq 29$ points (careful).

Physical activity was evaluated using a wristwatch-type accelerometer with the non-dominant hand for 72 hours (Life microscope; HitachiLtd, Tokyo, Japan). This instrument can detect changes in acceleration at a threshold of $0.01 \mathrm{G} / \mathrm{Rad} / \mathrm{s}$ in the range of 2 to $3 \mathrm{~Hz}$, count the number of times it exceeds, and record the number of changes in acceleration per minute. Sleep was evaluated based on five indicators: meandiurnal activity (DA), naps, total sleep time (TST), awakening $(\mathrm{Aw})$, and sleep efficiency (SE) [11].

\subsection{Data Collection Method}

In 2012, Autonomic nervous function was evaluated 1 hour from 9:00 before the start of chemotherapy. The autonomic nervous function in 2013 and 2014, when treatment was over, was also evaluated between 09:00 and 10:00. The subjective assessment of fatigue was evaluated by collecting the questionnaire by mail. As for the evaluation of physical activity, we had them wear a wristwatch-type accelerometer for 3 days. Only in 2012, to minimize the effects of side effects of chemotherapy drugs, the measurement date was 7 days after the start of treatment.

\subsection{Analysis Method}

The LF, HF, and LF/HF components were quantified and then logarithmized by 
heart rate variability analysis using an accelerometer. Data from 2012, 2013, and 2014 measurements were subjected to Pair-wise comparison of Friedman's test. Fisher's exact test was used for comparisons of subjective fatigue. DA, naps, TST, Aw, and SE were calculated to evaluate physical activity using analysis software. The level of significance for all tests was set at $5 \%$.

\subsection{Ethical Considerations}

This study was conducted with the cooperation of the director of the general hospital where the participants visited and the director of the nursing department, and was approved by the research ethics committee of the cooperating hospital. Approved by South Miyagi Medical Center (Number 2013-007).

\section{Results}

From 2012 to 2014, significant differences were found in physical fatigue ( $\mathrm{p}=$ $0.004)$, mental fatigue ( $p=0.002)$, comprehensive fatigue $(p=0.012)$, and comprehensive evaluations $(\mathrm{p}=0.032$ ). Data improved from 2012 to 2013 (physical fatigue: $\mathrm{p}=0.001$, mental fatigue: $\mathrm{p}=0.001$, comprehensive fatigue: $\mathrm{p}=0.008$ ), but again from 2013 to 2014 (physical fatigue: $\mathrm{p}=0.006$, mental fatigue: $\mathrm{p}=$ 0.002 , comprehensive fatigue: $\mathrm{p}=0.009$ ) the data was worse. Participant fatigues are shown in Table 2.

Similar to Subjective fatigue, there was a significant difference in the autonomic nervous function between the 3 groups from 2012 to 2014 (logLF: $\mathrm{p}=$ 0.017], $\log H F: p=0.023$ and $\operatorname{logLF} / H F: p=0.039$ ). It is linked to the results of Subjective fatigue, and the data improved from 2012 to 2013 (logLF: $p=0.001$, $\operatorname{logHF}: \mathrm{p}=0.019, \operatorname{logLF} / \mathrm{HF}: \mathrm{p}=0.018$ ), but deteriorated from 2013 to 2014 $(\log L F: p=0.008, \log H F: p=0.001, \log L F / H F: p=0.026)$. Evaluation of autonomic nervous function is shown in Table 3.

Table 2. Participants fatigue $(n=13)$.

\begin{tabular}{|c|c|c|c|c|c|}
\hline \multirow{2}{*}{ Item } & & 2012 & 2013 & 2014 & \multirow{2}{*}{ pvalue $^{\dagger}$} \\
\hline & & \multicolumn{3}{|c|}{ Mean $\pm \mathrm{SD} / \mathrm{n}(\%)$} & \\
\hline physical fatigue & & $10.31 \pm 2.95$ & $8.56 \pm 2.43$ & $9.31 \pm 2.82$ & 0.004 \\
\hline mental fatigue & & $12.34 \pm 3.18$ & $9.12 \pm 3.71$ & $10.57 \pm 3.05$ & 0.009 \\
\hline comprehensive fatigue & & $22.83 \pm 6.17$ & $17.92 \pm 5.47$ & $19.34 \pm 6.92$ & 0.012 \\
\hline \multirow{3}{*}{$\begin{array}{l}\text { Comprehensive } \\
\text { evaluation }\end{array}$} & good & $6(46.2)$ & $8(61.5)$ & $7(53.8)$ & \multirow{3}{*}{0.032} \\
\hline & attention & $4(23.1)$ & $4(30.8)$ & $3(23.1)$ & \\
\hline & careful & $3(23.1)$ & $1(7.7)$ & $3(23.1)$ & \\
\hline \multirow{2}{*}{\multicolumn{2}{|c|}{ Item }} & 2012 vs 2013 & 2013 vs 2014 & 2012 vs 2014 & \\
\hline & & \multicolumn{3}{|c|}{ Pair-wise comparison: $\mathrm{p}$ value $\mathrm{e}^{\alpha}$} & \\
\hline physical fatigue & & 0.001 & 0.006 & 0.005 & \\
\hline mental fatigue & & 0.001 & 0.002 & 0.003 & \\
\hline comprehensive fatigue & & 0.008 & 0.009 & 0.006 & \\
\hline
\end{tabular}

${ }^{\dagger}$ : Friedman's test, Fisher's exact test, ${ }^{a}$ : Bonferroni. 
Table 3. Evaluation of autonomic nervous function $(n=13)$.

\begin{tabular}{|c|c|c|c|c|}
\hline \multirow{2}{*}{ Item } & 2012 & 2013 & 2014 & \multirow{2}{*}{ p value } \\
\hline & \multicolumn{3}{|c|}{ mean $\pm \mathrm{SD}$} & \\
\hline $\log L F$ & $2.67 \pm 1.37$ & $2.12 \pm 1.17$ & $2.29 \pm 0.92$ & 0.017 \\
\hline $\log \mathrm{HF}$ & $2.36 \pm 1.59$ & $2.47 \pm 1.86$ & $2.25 \pm 1.27$ & 0.023 \\
\hline $\log L F / H F$ & $0.31 \pm 0.14$ & $0.19 \pm 0.11$ & $0.22 \pm 0.17$ & 0.039 \\
\hline \multirow{2}{*}{ Item } & 2012 vs 2013 & 2013 vs 2014 & 2012 vs 2014 & \\
\hline & \multicolumn{3}{|c|}{ Pair-wise comparison: $\mathrm{p}$ value ${ }^{\alpha}$} & \\
\hline $\log L F$ & 0.001 & 0.008 & 0.006 & \\
\hline $\log \mathrm{HF}$ & 0.019 & 0.001 & 0.013 & \\
\hline $\log \mathrm{LF} / \mathrm{HF}$ & 0.018 & 0.026 & 0.021 & \\
\hline
\end{tabular}

${ }^{\dagger}$ : Friedman's test, ${ }^{\alpha}$ : Bonferroni.

Similar to Subjective fatigue, there was a significant difference in the Physical activity between the 3 groups from 2012 to 2014 (DA [ $p=0.042$ ], TST [ $p=$ 0.037], SE [p = 0.018], AW [p = 0.023] and Naps [p=0.014]). It is linked to the results of Subjective fatigue, and the data improved from 2012 to 2013 (DA [p = $0.015]$, TST $[p=0.005]$, SE $[p=0.011]$, AW $[p=0.008]$ and Naps $[p=0.005])$, but deteriorated from 2013 to 2014 (DA [p $=0.021$ ], TST [p $=0.016$ ], SE [p = 0.017 , AW [ $=0.019]$ and Naps $[p=0.011])$. Physical activity evaluation is shown in Table 4.

\section{Discussion}

The study participants were cancer patients who were being treated in an outpatient clinic using a combination of multiple anticancer drugs. Most of the participants completed treatment in 2012, and in 2014, there was only one oral treatment. It is speculated that the side effects of chemotherapy were basically reduced over time. The participant's physical condition was in the range of PS 0 - 2 and had mild symptoms. Half of the participants were forced to move to temporary housing due to the Great East Japan Earthquake, and the community environment of some participants changed significantly compared to before the earthquake. Previous studies have also shown the relationship between local communities and mental health during disasters. Previous studies have also shown the relationship between local communities and mental health during disasters [12]. It is presumed that the victims were able to bring about mental well-being and reduce their fatigue if they felt the connection between the people around them and the community was active in the area.

Over time, the number of residents in temporary housing has decreased to two in 2014, reconstruction is progressing, and the living environment is improving. In the environment affected by the great disaster, the subjects speculate that the formation of a new community led to the reduction of physical distress.

It is also presumed that the participants also had less fatigue due to the sense of security that the treatment was completed. It is thought that the participant's 
Table 4. Physical activity evaluation $(\mathrm{n}=13)$.

\begin{tabular}{|c|c|c|c|c|}
\hline \multirow{2}{*}{ Item } & 2012 & 2013 & 2014 & \multirow{2}{*}{$\mathrm{p}$ value } \\
\hline & \multicolumn{3}{|c|}{ mean $\pm S D$} & \\
\hline DA & $1.7 \pm 0.7$ & $1.5 \pm 0.8$ & $1.6 \pm 0.5$ & 0.042 \\
\hline TST (Minute) & $470.2 \pm 58.1$ & $426.7 \pm 63.1$ & $446.9 \pm 52.6$ & 0.037 \\
\hline SE (\%) & $91.9 \pm 4.3$ & $94.8 \pm 2.9$ & $93.1 \pm 3.8$ & 0.038 \\
\hline Aw (Times) & $10.4 \pm 4.6$ & $6.5 \pm 3.8$ & $8.2 \pm 3.1$ & 0.023 \\
\hline Naps (Times) & $9.2 \pm 4.9$ & $5.4 \pm 3.3$ & $6.1 \pm 3.6$ & 0.014 \\
\hline \multirow{2}{*}{ Item } & 2012 vs 2013 & 2013 vs 2014 & 2012 vs 2014 & \\
\hline & \multicolumn{3}{|c|}{ Pair-wise comparison: $\mathrm{p}$ value ${ }^{\alpha}$} & \\
\hline DA & 0.015 & 0.021 & 0.019 & \\
\hline TST (Minute) & 0.005 & 0.016 & 0.016 & \\
\hline SE (\%) & 0.011 & 0.017 & 0.018 & \\
\hline Aw (Times) & 0.008 & 0.019 & 0.054 & \\
\hline Naps (Times) & 0.005 & 0.011 & 0.007 & \\
\hline
\end{tabular}

${ }^{\dagger}$ : Friedman's test, ${ }^{\alpha}$ : Bonferroni. DA: diurnal activity, TST: total sleep time, SE: sleep efficiency, AW: awakening.

ability to self-care against side effects improved with repeated treatment. However, there was a tendency for subjective fatigue to worsen in 2014 compared to the previous year. It is presumed that the background of these factors is not the complications caused by treatment but the change in life associated with reconstruction. Among the temporary housing, a new community was formed, but reconstruction housing was built around 2014, and it is necessary to reform the community. Survivors of natural disasters are at higher risk of social isolation due to the death of family or friends, and evacuation from their home community [13]. We think that those stresses are one of the causes. Participants were aware of fatigue caused by anticancer drugs, but it is presumed that they could be readjusted by repeating their experience as cancer survivors. Their PS was good and they were able to perform activities of daily living independently. Therefore, it is considered that the participants took appropriate coping actions to minimize the impact of the disaster. However, since cancer patients who have lost their homes due to the disaster may be socially isolated, it is important to provide psychological support.

Evaluations of physical activity and autonomic function showed significant differences over time. It is inferred that these changes are directly affected by the stress caused by the Great East Japan Earthquake. Stress affects the balance of the autonomic nervous system [14] [15]. Compared with the results of previous studies, the participant's $\log \mathrm{LF} / \mathrm{HF}$ was an active sympathetic nervous system. In this study, surveys were conducted 1.5, 2.5, and 3.5 years after the earthquake. The recovery period to overcome an acute disaster is an activity such as removing debris and moving to temporary housing. However, the living environment of temporary housing affects autonomic nervous function because it causes 
stress due to problems such as noise from thin walls, room temperature, and the construction of new communities. In 2014, many people are moving from temporary housing to Public reconstruction housing. Their new surroundings require them to rebuild personal relationships and to adapt to a different environment [13]. Experiencing stress from both cancer treatment and the earthquake is considered to have a greater effect on the sympathetic nervous system than is cancer treatment alone.

Most of the participants in this study were older than 65 years, but the measurements were higher than the logHF (standard: 1.50) of healthy individuals. The plasma levels of noradrenaline and adrenalin have a 24-h circadian rhythm, with adrenalin at a minimum around 03:00 before increasing sharply to a peak around 09:00. As a generalized response to physical and mental stress, the autonomic nerves are affected, the sympathetic nervous system is activated, and the parasympathetic nerves are suppressed [7] [16] [17]. As the LF and HF components decrease with age, the power value also decreases because of the reduced thickening of the arterial wall and the increased pressure due to vasoconstriction [18] [19]. In particular, the HF component, which reflects parasympathetic nerve function, is reported to decrease rapidly with age and to exacerbate fatigue [4]. In 2013 and 2014, the number of subjects who completed treatment increased, but we think that aging and changes in living environment affected the autonomic nerves.

In this study, participants' autonomic function was evaluated immediately after the start of chemotherapy after an outpatient examination. The measurement time was from 10:00 to 12:00. Therefore, considering the physiological response of the participants' plasma concentration and psychological state immediately before chemotherapy, an increase in LF value could be predicted, and the balance of LF/HF ratio could be disturbed. To maintain homeostasis, not only the autonomic nervous system, but also regulatory functions such as the endocrine and immune systems, need to be in a state of allostasis [20]. Nurses observe the general condition of the cancer patient for evidence of side effects of the anticancer drug. In addition, nurses ask such patients questions such as work, family, and living background to better understand their perception of changes in their living environment. It is important for care professionals to listen to complaints about anxiety and pain in cancer patients, collaborate across different occupations, and build an environment in which they can support. It is also important to prevent exacerbation of cardiovascular disease, death from overwork, and sudden death while paying attention to mental and physical changes and medical history other than cancer treatment.

The results of the physical activity evaluations showed that the patients' rest and activity time changed in accordance with changes in autonomic nervous function. The first measured sleep time was about $470 \mathrm{~min}$. Focusing only on this 470-minute sleep time, it is safe to judge that the sleep condition is good. However, sleeping efficiency was very poor (SE $=91.9 \%)$, and more than ten halfway awakenings were recorded. Participants spend more time sleeping and 
taking naps during the day when they should be active, which is presumed to have led to an increase in overall sleep time. It is said that stress before sleep onset affects autonomic nervous function during sleep [21] [22]. In general, fatigue is experienced by everyone on a daily basis, and although there are individual differences, the symptoms of resting and sleeping at night are alleviated. The sleep cycle also changed according to the change in subjective evaluation. There is also a risk of becoming alexisomia in a highly stressed environment [23]. Since alexisomia is in a state where subjective symptom recognition and objective symptom do not match, it is important to comprehensively evaluate the participants.

\section{Direction of Nursing}

This study is a three-year cross-sectional study by the same participants. However, for autonomic nervous function and sleep, it is necessary to calculate the baseline from the daily measurement results and judge the meaning of the change. Moreover, the fact that the number of participants was as small as 13 was the limit of this study. The simple measuring instrument used in this study can quantify subjective fatigue with objective numerical values and apply it to individualized care. Autonomic nervous function is very sensitive to chemotherapy and side effects, and sleep disorders are widespread. The aim of care professionals is to analyze the objective rationale behind the patient's subjective data and to systematically educate and coordinate the patient's overall daily life.

It is also important for cancer patients to continue measurement from normal times over time, rather than evaluating fatigue according to their physical and mental condition using objective indicators only when a disaster occurs. In addition, if treatment cannot be continued due to damage caused by a disaster, it is necessary to refer to medical areas and hospitals other than the disaster area while sharing patient information using ICT technology.

\section{Conclusion}

The study participants consisted of 13 cancer outpatients. The participants fatigue worsened at one time, but showed improvement over time. The role of the care professional is to understand the patient's subjective and objective data and to systematically understand the patient's daily life. In the event of an emergency such as a disaster, it is necessary to evaluate the physical and mental condition based on objective indicators of cancer patients receiving anti-cancer drug treatment and provide nursing support.

\section{Financial Support and Sponsorship}

This study was supported by The Yasuda Medical Foundation (Cancer Nursing Research Grant 2013).

\section{Conflicts of Interest}

The author declares no conflicts of interest. 


\section{References}

[1] Kondo, S., Shiba, S., Udagawa, R., Ryushima, Y., Yano, M., Uehara, T., et al. (2015) Assessment of Adverse Events via a Telephone Consultation Service for Cancer Patients Receiving Ambulatory Chemotherapy. BMC Research Notes, 8, 315. https://doi.org/10.1186/s13104-015-1292-8

[2] Sato, D. (2020) Evaluation of Fatigue in Cancer Patients in an Area Affected by the Great East Japan Earthquake. Asia-Pacific Journal of Oncology Nursing, 7, 203-208. https://doi.org/10.4103/apjon.apjon_52_19

[3] Atsushi, S., Masaaki, T. and Toshitaka, R. (2013) Survey of Mental Health Support Activities for Disaster Relief Teams and Residual Members in the Great East Japan Earthquake. Annual Research Report of JSDF Sapporo General Hospital, 51, 47-51. (In Japanese)

[4] Takashi, W., Toshinori, S., Toru, T., Yasutake, E., Yumi, S., Msateru, K., et al. (2013) Changes in Subjective Symptom Rate before and after the Great East Japan Earthquake. Annual Health, Labour and Welfare Report, 60, 1-6. (In Japanese)

[5] Yilmaz, V. (2004) A Statistical Analysis of the Effects on Survivors of the 1999 Earthquake in Turkey. Social Behavior and Personality, 32, 551-558. https://doi.org/10.2224/sbp.2004.32.6.551

[6] Mizuno, K., Tanaka, M., Yamaguti, K., Kajimoto, O., Kuratsune, H. and Watanabe, Y. (2011) Mental Fatigue Caused by Prolonged Cognitive Load Associated with Sympathetic Hyperactivity. Behavioral and Brain Functions, 7, 17. https://doi.org/10.1186/1744-9081-7-17

[7] Kuratsune, H. (2011) Establishment of Objective Fatigue Diagnosis Method and Creation of Chronic Fatigue Diagnosis Guidelines for Patients Complaining of Chronic Fatigue with Autonomic Dysfunction. Health Labour Sciences Research Grant Research Report 2011, 1-19. (In Japanese)

[8] Tanaka, M., Mizuno, K., Yamaguti, K., Kuratsune, H., Fujii, A., Baba, H., Matsuda, K., Nishimae, A., Takesaka, T. and Watanabe, Y. (2011) Autonomic Nervous Alterations Associated with Daily Level of Fatigue. Behavioral and Brain Functions, 7, Article No. 46. https://doi.org/10.1186/1744-9081-7-46

[9] Mizuno, K., Tajima, K., Watanabe, Y. and Kuratsune, H. (2014) Fatigue Correlates with the Decrease in Parasympathetic Sinus Modulation Induced by a Cognitive Challenge. Behavioral and Brain Functions, 10, 25. https://doi.org/10.1186/1744-9081-10-25

[10] Lázaro, J., Gil, E., Orini, M., Laguna, P. and Bailón, R. (2019) Baroreflex Sensitivity Measured by Pulse Photoplethysmography. Frontiers in Neuroscience, 13, 339. https://doi.org/10.3389/fnins.2019.00339

[11] Morgenthaler, T., Alessi, C., Friedman, L., Owens, J. and Kapur, V. (2007) Practice Parameters for the Use of Actigraphy in the Assessment of Sleep and Sleep Disorders: An Update for 2007. Sleep, 30, 519-529. https://doi.org/10.1093/sleep/30.4.519

[12] Kino, S., Aida, J., Kondo, K. and Kawachi, I. (2020) Long-Term Trends in Mental Health Disorders after the 2011 Great East Japan Earthquake and Tsunami. JAMA Network Open, 3, e2013437. https://doi.org/10.1001/jamanetworkopen.2020.13437

[13] Takuya, S., Yoshihiro, H. and Yumi, S. (2019) Moving from Prefabricated Temporary Housing to Public Reconstruction Housing and Social Isolation after the Great East Japan Earthquake: A Longitudinal Study Using Propensity Score Matching. BMJ Open, 9, e026354. https://doi.org/10.1136/bmjopen-2018-026354

[14] Abboud, F.M. and Singh, M.V. (2017) Autonomic Regulation of the Immune Sys- 
tem in Cardiovascular Diseases. Advances in Physiology Education, 41, 578-593. https://doi.org/10.1152/advan.00061.2017

[15] Vaillancourt, M., Chia, P., Sarji, S., Nguyen, J., Hoftman, N., Ruffenach, G., et al. (2017) Autonomic Nervous System Involvement in Pulmonary Arterial Hypertension. Respiratory Research, 18, Article No. 201.

https://doi.org/10.1186/s12931-017-0679-6

[16] Langewitz, W., Rüddel, H., Schächinger, H., Lepper, W., Mulder, L.J. and Veldman, J.H. (1991) Changes in Sympathetic and Parasympathetic Cardiac Activation during Mental Load: An Assessment by Spectral Analysis of Heart Rate Variability. Homeostasis in Health and Disease, 33, 23-33.

[17] Wang, X., Liu, B., Xie, L., Yu, X., Li, M., Zhang, J., et al. (2016) Cerebral and Neural Regulation of Cardiovascular Activity during Mental Stress. BioMedical Engineering OnLine, 15, 160. https://doi.org/10.1186/s12938-016-0255-1

[18] Tobaldini, E., Toschi-Dias, E., Appratto de Souza, L., RabelloCasali, K., Vicenzi, M., Sandrone, G., et al. (2019) Cardiac and Peripheral Autonomic Responses to Orthostatic Stress during Transcutaneous Vagus Nerve Stimulation in Healthy Subjects. Journal of Clinical Medicine, 8, 496. https://doi.org/10.3390/jcm8040496

[19] Yamaki, F., Obara, K. and Tanaka, Y. (2019) Angiotensin II Regulates Excitability and Contractile Functions of Myocardium and Smooth Muscles through Autonomic Nervous Transmission. Yakugaku Zasshi, 139, 793-805. (In Japanese) https://doi.org/10.1248/yakushi.19-00002

[20] Ramsay, D.S. and Woods, S.C. (2014) Clarifying the Roles of Homeostasis and Allostasis in Physiological Regulation. Psychological Review, 121, 225-247. https://doi.org/10.1037/a0035942

[21] Cole, R.J., Kripke, D.F., Gruen, W., Mullaney, D.J. and Gillin, J.C. (1992) Automatic Sleep/Wake Identification from Wrist Activity. Sleep, 15, 461-469. https://doi.org/10.1093/sleep/15.5.461

[22] Hall, M., Vasko, R., Buysse, D., Ombao, H., Chen, Q., Cashmere, J.D., et al. (2004) Acute Stress Affects Heart Rate Variability during Sleep. Psychosomatic Medicine, 66, 56-62. https://doi.org/10.1097/01.PSY.0000106884.58744.09

[23] Kenji, K., Yuko, M., Mikihiko, F., Shinichiro, I. and Naoki, T. (2004) Paradoxical Results of Psychophysiological Stress Profile in Functional Somatic Syndrome: Correlation between Subjective Tension Score and Objective Stress Response. Applied Psychophysiology and Biofeedback, 29, 255-268. https://doi.org/10.1007/s10484-004-0386-1 